\title{
Algunas consideraciones sobre las transformaciones actuales de las finanzas locales en Francia
}

\section{Some considerations on the current transformations of local finance in France}

\author{
Marcela Astudillo-Moya*
}

\begin{abstract}
In the analysis of the current transformations of local finance in France this paper highlights the importance of local public administration. Based on the hypotheticaldeductive method, it concludes that one of the challenges faced by the future of local finance in France is to ensure a steady growth in a cohesive territory, for which a distribution of tax sources coordinated with the central government is required, so that local authorities have decision autonomy.
\end{abstract}

Keywords: local finance, public expenditure, public revenue, urban development, fiscal centralization.

\section{Resumen}

En el análisis de las transformaciones actuales de las finanzas locales en Francia, realizado en este artículo, destaca la importancia de la administración pública local. Con base en el método hipotético-deductivo se concluye que uno de los desafíos a los que debe hacer frente el futuro de la hacienda local en Francia es el de garantizar un crecimiento estable en un territorio cohesionado, para lo que se requiere de una distribución de fuentes impositivas coordinada con la administración pública central, de tal manera que las colectividades locales cuenten con autonomía de decisión.

Palabras clave: finanzas locales, gasto público, ingreso público, desarrollo urbano, centralización fiscal.

* Universidad Nacional Autónoma de México. Correo-e: marcelaa@unam.mx

La autora agradece a la DGAPA-UNAM su valioso apoyo para realizar una estancia sabática de investigación en la Universidad Jean Moulin Lyon 3 de Francia. 


\section{Introducción}

El objetivo de esta investigación es analizar la evolución reciente de las finanzas locales en Francia, Estado unitario que ha iniciado el reconocimiento de la esfera local a través de un proceso de descentralización y que durante los últimos 30 años ha presentado cambios permanentes dirigidos a profundizar este proceso. Las cuestiones a resolver a lo largo de este trabajo son ¿qué importancia tienen las finanzas locales en las finanzas públicas francesas? ¿este país enfrenta actualmente una crisis de las finanzas locales? ¿cuáles son los nuevos objetivos asignados a las finanzas de las colectividades territoriales? ¿cuál es el fundamento fiscal del principio de la libre administración de las colectividades locales? ¿qué escenarios se plantean para el futuro de las finanzas locales francesas?

Para resolver estas cuestiones se utiliza el método hipotético-deductivo cuyo punto de partida está constituido por los supuestos de la teoría de las finanzas locales, tomados como criterios de elección de las variables que integran el modelo planteado como escenarios del futuro de las finanzas locales, el cual se buscará comprobar lógicamente.

En el primer apartado de este trabajo se estudian las características de las finanzas locales francesas, en el segundo se analizan las transformaciones de la gestión financiera local, y en el último apartado se revisan algunos escenarios del futuro de las finanzas locales. Finalmente, se concluye que para garantizar un crecimiento estable en un territorio cohesionado se requiere la coordinación de los diferentes niveles de gobierno, de tal manera que las colectividades locales cuenten con autonomía de decisión.

\section{1. ¿Crisis de las finanzas locales?}

Para explicar la estructura, así como la coyuntura, y determinar el posible modelo futuro que tome en cuenta la especificidad de las finanzas públicas francesas, el estudio se enfoca principalmente al análisis de la tendencia del comportamiento de las haciendas locales durante un periodo de 30 años.

Resulta importante señalar que las finanzas locales fueron consideradas secundarias por largo tiempo. A finales del siglo XIX (1884) se establecía escuetamente en la legislación que el consejo municipal tenía por función regular, a través de deliberaciones, los asuntos de los municipios sin precisar la naturaleza de los asuntos, lo cual, explican algunos autores (Cabannes, 2007; Buisson, 2009), se debía a que la vida municipal se reducía a la gestión del patrimonio, a la seguridad vecinal, a la enseñanza y al cuidado de los bosques. Así, las funciones del consejo municipal se cir- 
cunscribían a emitir algunos reglamentos y votar un presupuesto, así como organizar fiestas y ceremonias.

La evolución ulterior confirma un incremento inexorable de los gastos locales, así como de las funciones a cargo de los municipios derivadas de la intervención creciente de las colectividades locales (y del sector público en general) en el terreno económico y social; de esta manera, las municipalidades tomaron directamente a su cargo ciertas necesidades públicas que el sector privado no podía seguir satisfaciendo.

La necesidad de reconstruir al país después de la Primera Guerra Mundial, así como la crisis económica de 1929, es un elemento decisivo que obliga al sector local a realizar un número muy importante de equipamientos colectivos (Brunet-Léchenault, 2000).

Después de la Segunda Guerra Mundial, en concordancia con las teorías keynesianas que predominaban entonces, la expansión del Estado de bienestar discurre en paralelo al aumento muy apreciable de peso de los gobiernos locales. El Estado delega progresivamente a las colectividades territoriales un número mayor de tareas. Esta etapa está caracterizada por la fuerte expansión del gasto público local, consecuencia del aumento de necesidades de servicios locales, tanto por el crecimiento de su demanda como del costo real de operación (Greffe, 2005). Por esto los gastos locales de inversión y, por lo tanto de funcionamiento, se incrementan considerablemente. ${ }^{1}$ Asimismo, se observa una demanda de infraestructura que se incrementa incesantemente como consecuencia de la aparición de nuevas necesidades ligadas a la expansión industrial y un modo de financiamiento inadecuado.

${ }^{1}$ El presupuesto de gastos consta de dos grandes rubros: la sección de funcionamiento y la de inversión. En la primera se encuentran todos los gastos necesarios para el funcionamiento de los servicios de las colectividades que son regulares de cada año: remuneraciones de personal, materiales y consumos corrientes, pequeños gastos de conservación, participaciones y cargos de organismos exteriores (ayuda social, sindicatos intermunicipales) y el pago de intereses de la deuda.

En contraparte, la sección de funcionamiento se financia con recursos en principio regulares, como son los productos de explotación de sus propiedades, de subvenciones, dotaciones y participaciones del Estado o de otras colectividades y, desde luego, se incluyen los ingresos fiscales provenientes especialmente de los cuatro grandes impuestos directos.

En la segunda sección se incluyen los gastos de inversión, los cuales se aplican esencialmente a las operaciones no repetitivas, es decir, que no se renuevan cada año y que se traducen en una modificación apreciable o enriquecimiento del patrimonio de la colectividad: compra de materiales durables, construcción o ampliación de construcciones existentes, pago de subvenciones de equipamiento. Comprende también las amortizaciones de la deuda.

El financiamiento de esta sección está asegurada por los recursos definitivos que recibe la colectividad y por los préstamos que deberá reembolsar en el futuro.

Es importante subrayar que los gastos de inversión generan en el futuro gastos de funcionamiento por mantenimiento o de personal. Por ejemplo, un equipo de incineración de basura, aunque esté automatizado, requiere de la intervención permanente de personal.

Los recursos definitivos están constituidos fundamentalmente por dotaciones o subvenciones provenientes del Estado o de otras colectividades (departamento o región), de recursos obtenidos para un fin específico, de cesión de activos y de retención de los recursos corrientes de los municipios. 
A lo largo de esta evolución, los recursos de las colectividades territoriales se caracterizaron por la insuficiencia de sus ingresos fiscales y dieron lugar a tensiones financieras en el plano local (Teyssier, 2006), y a una crisis de las finanzas locales que se trató de remediar con una serie de reformas propuestas a principios del siglo xx; como se observa, el fenómeno no es realmente nuevo. Esta crisis financiera local tiene también sus raíces profundas en el papel que las colectividades locales son llamadas a ejercer dentro de la evolución general de la sociedad, lo cual ha generado problemas que no han podido ser remediados debido a la ineficacia técnica y al volumen de medios financieros de que disponen. Se puede observar, por consecuencia, no un problema reciente sino más bien una cuestión recurrente presentada en contextos diferentes según las épocas.

\subsection{El nuevo rol de las finanzas locales}

Se puede decir que la segunda mitad de los setenta se caracterizó por el incremento en el precio del petróleo y por una atención mayor a las estructuras y mecanismos financieros públicos debido a los problemas presupuestarios y financieros, así como por las controversias teóricas suscitadas por los efectos y dificultades del modelo de Estado benefactor. También el impulso de diferentes escuelas económicas, por ejemplo la del Public Choice de Chicago o la escuela de Friedrich Hayek de Austria, contribuyó a la renovación de tesis económicas liberales que sostienen la idea de sustituir las políticas keynesianas por una política de oferta que implica un gran movimiento de liberalización de la economía y, por tanto, una salida del Estado del mercado, así como por la adopción de un modelo de gestión del sector público a imagen de las empresas privadas.

Paralelamente, la liberalización significa también un redireccionamiento de los fondos públicos en el terreno económico (para apoyar a las empresas por la vía de incentivos fiscales) y por consecuencia una redefinición de las políticas públicas. El Estado igualmente preconiza que las colectividades territoriales adquieren una parte muy importante de responsabilidad en esta dinamización de corte económico y social (Greffe, 2005).

La descentralización se convierte también en uno de los conceptos importantes de las teorías económico liberales, ya que ellas buscan un campo de intervención económico susceptible de alcanzar la libertad de mercado. Las colectividades territoriales se consideran capaces de participar activamente, acompañar o coadyuvar al desarrollo económico. Esto dentro del mismo cuadro de análisis que considera a los administradores locales como los animadores económicos, con vocación a suscitar o catalizar las energías para impulsar un desarrollo económico local, campo en el que uno de los principales problemas es la localización de las empresas 
y la provisión de la infraestructura necesaria (Tulard, 2006). Así, los administradores locales pueden favorecer e incitar la implantación de establecimientos en un determinado territorio.

Esto se encuadra en un contexto de ideas que se orientan a una nueva función, al considerarse a las finanzas locales y más precisamente a la gestión de los recursos locales como un medio de primera importancia para generar el desarrollo económico local o para contribuir a su expansión (Gevart, 2006).

Así, es hasta un periodo reciente que se reconoce su papel relevante en las finanzas públicas. Hoy en día, las colectividades locales se han convertido en actores esenciales de las sociedades contemporáneas. Sus actividades se extienden a múltiples sectores de la vida colectiva y su campo de competencia es muy diverso: ordenación territorial, la lucha contra la exclusión, la enseñanza, los transportes, la cultura y otros (Boeuf y Magnan, 2009). Además de que la estructura del presupuesto tiene un efecto importante en la primera ronda de votación para la elección de autoridades municipales (Cassette et al., 2013).

Estadísticamente se puede observar que los gastos de la administración pública local representaron $52.7 \%$ de los gastos de la administración pública central en 2008, dicha participación ha mostrado una tendencia ascendente durante el periodo 1978-2008.

Como se observa en la gráfica 1 , el crecimiento de los gastos de la administración pública local fue mayor $(726.2 \%)$ que los correspondientes al gobierno central (318.6\%) en el periodo señalado anteriormente. ${ }^{2}$ Es importante señalar que este crecimiento se explica por los gastos derivados de la descentralización. En 1978 los gastos locales representaban $8 \%$ del piв y se incrementaron a $11 \%$ treinta años más tarde; pero si no se consideraran los gastos por la descentralización, representarían un poco menos de 2\% (Laurent, 2009).

Igualmente, los gobiernos locales desempeñan un papel clave en el conjunto de la inversión pública. Ello es indicativo del carácter de los servicios prestados por estos gobiernos y sirve para subrayar la importancia de la actividad local en términos macroeconómicos y de estabilización. En 1978 realizaron $59.1 \%$ de la formación bruta de capital fijo; mientras que en 2008 alcanzó la cifra de 76.7\%, resultado de las tendencias de crecimiento que se observan en la gráfica 2 (incremento de $587.8 \%$ durante el periodo 1978-2008).

\footnotetext{
${ }^{2}$ Cabe mencionar que, de acuerdo con el estudio de Facchini y Melki (2013), cuando el gasto del sector público representa alrededor de 30\% del piв se alcanza en Francia el tamańo de un gobierno eficiente.
} 


\section{Gráfica 1}

Gastos de la administración pública

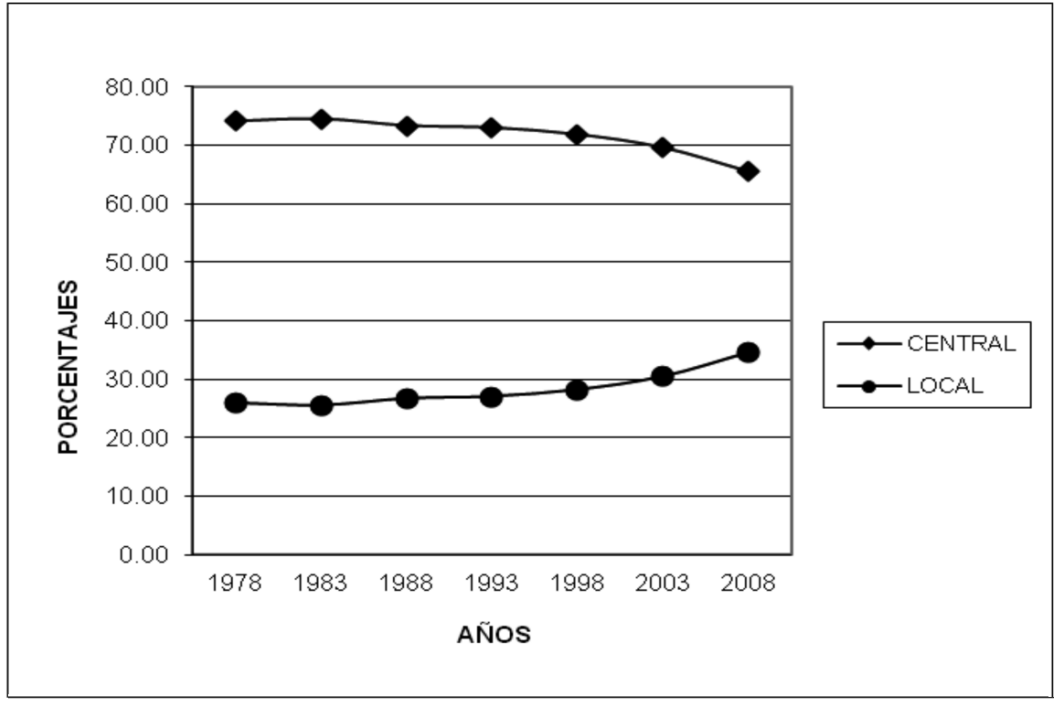

Base 2000

Fuente: INSÉÉ, 2009.

\section{Gráfica 2}

\section{Formación bruta de capital fijo}

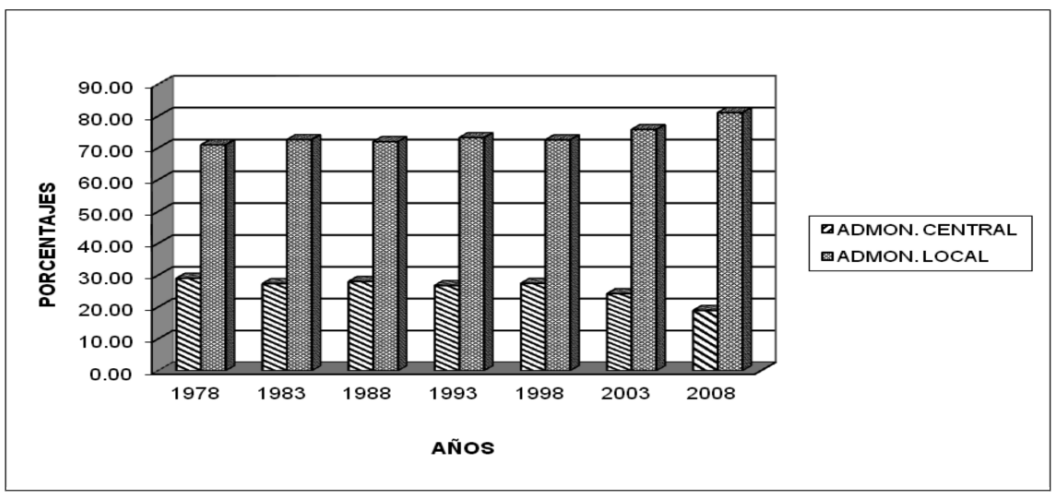

Base 2000

Fuente: INSÉÉ, 2009. 
Por otra parte, en la gráfica 3 se constata también que al igual que los gastos, los ingresos locales tienen cada ańo un peso mayor. En 2008 representaron $58.6 \%$ de los ingresos de la administración pública central; en 1978 significaban solamente $30.1 \%$.

\section{Gráfica 3}

\section{Ingresos de la administración pública}

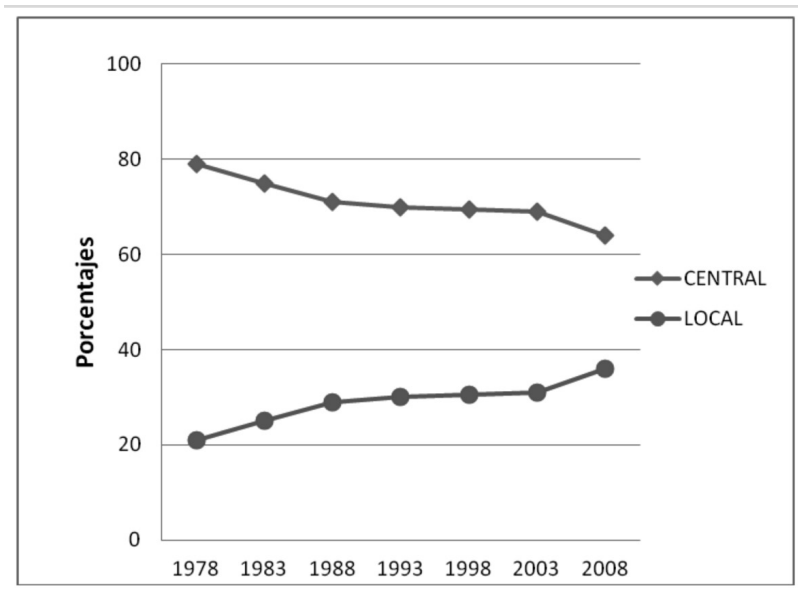

Base 2000

Fuente: INSÉÉ, 2009.

Así pues, pareciera inadecuado designar crisis de las finanzas locales a una situación financiera que globalmente presenta una evolución a la alza. Por ejemplo, las necesidades de financiamiento de la administración pública local durante el periodo 1978-2008 crecieron $115 \%$ como puede verse en la gráfica 4.

Es inevitable que las colectividades locales, al igual que numerosas instituciones públicas o privadas, tengan que enfrentar importantes cambios en su ambiente interno y externo que implican una renovación constante y por lo tanto una necesidad creciente de medios financieros.

\subsection{La disciplina presupuestaria, un elemento a considerar}

Sin duda, el fenómeno más importante dentro de las transformaciones de las finanzas públicas de los últimos 30 años es el desarrollo de una cultura financiera nueva, una cultura marcada por el liberalismo económico, el problema del incremento de los gastos públicos y, más aún, el de la racionalización de la gestión.

Esta cultura ha sido en gran parte inducida y se puede decir forzada por las normas y la lógica de la construcción de la Unión Europea (Au- 


\section{Gráfica 4 \\ Capacidad o necesidad de financiamiento de la administración pública local}

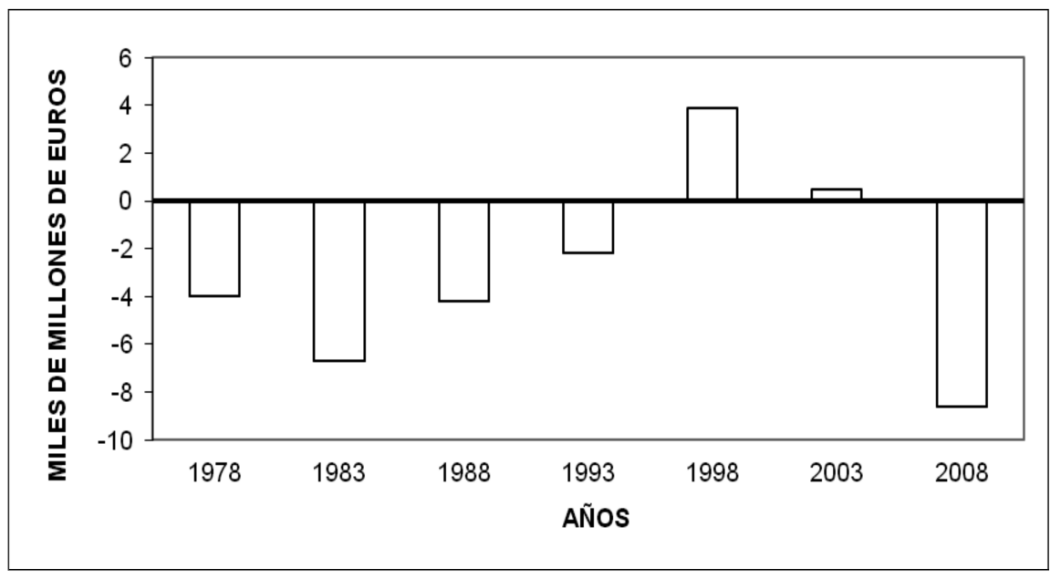

Base 2000

Fuente: INSÉÉ, 2009.

bouin y Moraud, 2006). Las transformaciones del sistema financiero público producidas en los últimos años han girado en torno a la unión económica y monetaria; fundamentada en la disciplina presupuestaria, lo que algunos autores denominan regla de oro (Fabbrini, 2013).

Esta disciplina se entiende, en primer lugar, respecto de un cierto número de criterios financieros tales como la limitación del endeudamiento público y del déficit público y, por supuesto, el equilibrio presupuestario; criterios que se relajaron a raíz de la crisis financiera del 2010 y que, en la actualidad, tienden a retomarse. En el caso francés, si bien el déficit de la administración pública local creció de manera importante en el periodo 1978-2008, como se observó en la gráfica 4, resulta que pasó de representar $17.6 \%$ del total de sus ingresos de 1978 a solamente $4.0 \%$ en 2008, como puede constatarse en la gráfica 5.

En términos generales, los gobiernos locales han utilizado la deuda para financiar un incremento real del patrimonio de las colectividades locales y en ningún caso para cubrir una insuficiencia de sus ingresos corrientes (Tulard, 2006).

En segundo lugar, la disciplina presupuestaria se basa en un enfoque global de las finanzas públicas entendidas como un todo que comprende las finanzas del Estado, de las colectividades locales y de los organismos de seguridad social. Este enfoque que no separa de manera absoluta los diferentes sectores de finanzas públicas conduce también a admitir que las compensaciones entre los diferentes componentes o el debilitamiento 


\section{Gráfica 5 \\ Administración pública local \\ Déficit en proporción al total de ingresos}

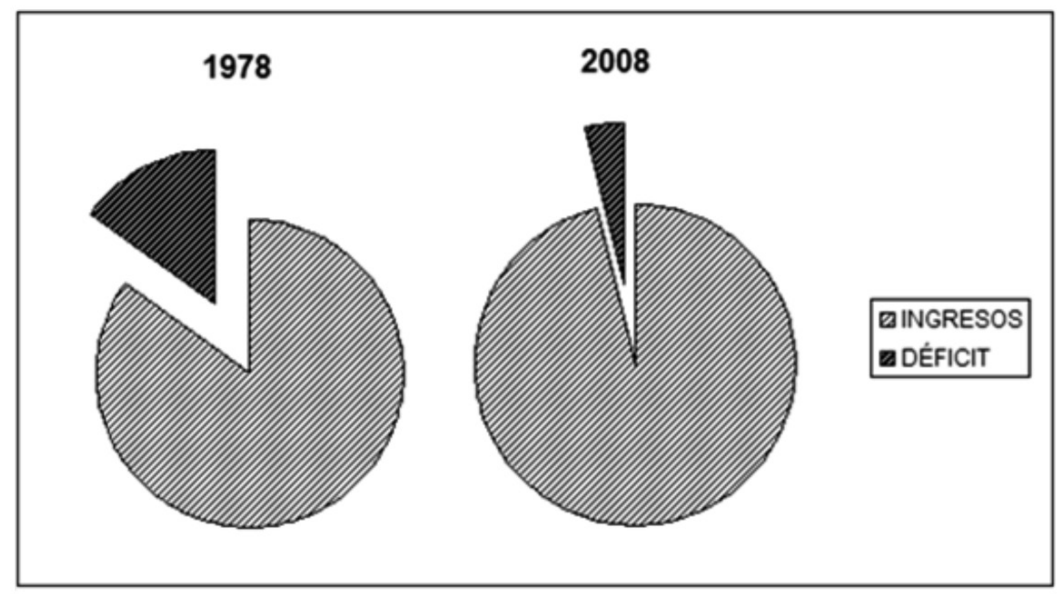

Base 2000

Fuente: INSEE, 2009.

de uno de ellos es susceptible de debilitar al conjunto interdependiente, por ejemplo, de impedir satisfacer los compromisos plurianuales derivados del Pacto de Estabilidad y Crecimiento.

Lo anterior, hace pensar inevitablemente en el peligro potencial de descontrol que pueden representar los millares de colectividades locales en las cuales las disparidades de riqueza, de población y de superficie, causan una gran diversidad de poder de decisión (Aubouin y Moraud, 2006).

A fin de responder a tal riesgo, que es calificado de riesgo sistémico, se ha propuesto instituir un control central que reduzca o suprima la autonomía financiera local, lo que remite inmediatamente a cuestionar la descentralización o por lo menos a otra concepción que distingue la autonomía de gestión de la autonomía de decisión. En cualquier caso, la problemática a resolver es de orden político, ya que concierne finalmente al equilibrio de poderes en el seno de los Estados pertenecientes a las regiones económicas y monetarias dotadas de un sistema de vigilancia multilateral, como es el caso de la Unión Europea (Bouvier, 2007).

\section{Las transformaciones de la gestión financiera local}

Tanto en la práctica como en la teoría hay una verdadera metamorfosis de la gestión financiera local francesa, fundamentalmente durante los últimos 30 años. La realidad financiera local, en la actualidad, desborda en gran medida los cuadros orgánicos tradicionales (Greffe, 2005) y se 
encuentra encerrada en una red de estructuras y de relaciones cada vez más complejas, más interrelacionadas, o sea dentro de un sistema interactivo compuesto de conjuntos y subconjuntos que están en un desarrollo continuo.

En Francia hay 36,808 colectividades territoriales (representan 40\% del total de la Unión Europea) que si bien garantizan una proximidad real de la acción pública a los ciudadanos, limitan la capacidad de intervención individual de los mismos. De ahí que se han desarrollado estructuras administrativas para lograr una gestión eficiente que aproveche las economías de escala y mejore la solidaridad financiera. Ejemplo de ellas son los Establecimientos Públicos de Cooperación Intermunicipal (EPCI) que pueden tener fiscalidad propia o carecer de ella.

En cualquier caso, su objetivo es asociar a los municipios en el seno de un espacio de solidaridad para la elaboración de un proyecto común, por ejemplo: desarrollo económico, gestión del ambiente, ordenación del territorio, vías de comunicación, equipamiento cultural y deportivo, alimentación de agua potable, electrificación u otros. ${ }^{3}$

Tal sistema confiere un enfoque nuevo a la gestión financiera local que necesariamente modifica la acción y las características de los poderes financieros locales (Faure y Doiullet, 2005). Sobre el terreno financiero y de gestión, esta reorganización del sistema local tiene nuevos problemas. La contraparte de tal realidad se expresa en primer lugar por las dificultades de la administración que se plantea debido a la obsolescencia de las reglas tradicionales de gestión del sector público.

\subsection{Objetivos asignados a las finanzas locales}

La noción de desarrollo económico local asociado a la de ordenación territorial, en la actualidad, es una parte integrante de los objetivos asignados a las finanzas locales. Tradicionalmente se consideraban funciones relevantes de la administración pública central, pero se han convertido, al menos para algunas colectividades territoriales francesas, en parte de sus funciones (Albert y Saïdj, 2009).

La noción de desarrollo económico local y las prácticas que la ilustran (que hacen su debut en los ańos ochenta en un contexto marcado por el

\footnotetext{
${ }^{3}$ Los EPCI con fiscalidad propia pueden ser comunidad de municipios, comunidad de aglomeración o comunidad urbana; cada categoría depende del número de habitantes y cada una de ellas tiene sus propias funciones a desarrollar.

Los EPCI sin fiscalidad propia pueden ser sindicatos de vocación única (Sivu), sindicatos de vocación múltiple (Sivom) o sindicatos mixtos. En este caso los municipios se asocian para realizar obras de interés común. Para la creación de un sindicato no es importante el número de habitantes. Los municipios pueden pertenecer al mismo o a diferentes departamentos y pueden ser o no limítrofes. Son creados sin tener una fecha fija de terminación.
} 
lanzamiento de la dinámica descentralizadora y la preocupación de remediar los fenómenos de desindustrialización) hacen aparecer la concepción de un desarrollo endógeno y ascendente ${ }^{4}$ que se opone a la concepción de un desarrollo exógeno y descendente.

Posteriormente aparece el enfoque transversal de desarrollo (entendido como un desarrollo global que supera la distinción entre desarrollo ascendente o descendente), que ha terminado por prevalecer y que apuntala la idea de un desarrollo que descansa en la colaboración del sector privado y el sector público (Gouttebel, 2010). El desarrollo desde este enfoque se basa fundamentalmente en un conjunto de colaboradores, potenciales o declarados, susceptibles de generar el desarrollo económico en un espacio local determinado, se entiende que cada uno de ellos conserva sus intereses propios, pero se integran en una estrategia global portadora de una dinámica favorable a todos.

Es el caso de los sindicatos mixtos que reúnen colectividades territoriales de niveles diferentes (municipios, departamentos o regiones) y diversas personas morales de derecho público, cámaras de comercio, de industria y de agricultura, para realizar obras o servicios de interés común. Es usual considerar una colaboración pública-privada, con la nueva herramienta jurídica denominada contrato de colaboración, el cual considera que dos iguales encuentran cada uno ventajas para ponerse de acuerdo respecto a un objeto común. Los criterios para recurrir a un contrato de colaboración según la ley son la urgencia y la complejidad (Laurent, 2009).

Bajo esta lógica, las modificaciones al marco normativo de 1983 plantean la extensión del campo de competencia de las colectividades territoriales, se considera que los municipios, departamentos y regiones son lugares donde se puede presentar la interrelación del sector público y del privado. Es innegable que todos, fundamentalmente los municipios, no pueden cumplir el mismo papel debido a las diferencias de tamaño: alrededor de 32,000 municipios cuenta con menos de 2,000 habitantes y de éstos, 4,000 tiene una población inferior a 100 habitantes; mientras que aproximadamente 900 tiene más de 10,000 habitantes (Laurent, 2009).

Además, presentan diferencias por su situación geográfica (por ejemplo, zonas de montańa, litorales), así como en los problemas más o menos agudos que les afectan. Esta diversidad de territorios hace que la única

\footnotetext{
${ }^{4}$ La concepción de que las colectividades territoriales son consideradas susceptibles de favorecer las condiciones de un crecimiento económico al desarrollar las potencialidades de su territorio ha sido sostenida tanto por algunos economistas como urbanistas. Small is beautiful es el lema que difundió E. Schumacher, quien junto con otros teóricos valorizan el rol esencial en el crecimiento económico de las pequeñas y medianas empresas, ya que son más dinámicas que las grandes empresas, por lo tanto tienen una mayor capacidad de crear empleos, son la puerta de un crecimiento económico (Bouvier et al., 2008).
} 
respuesta posible sea la diversidad de políticas locales; sin embargo, en todos los programas que emanan de las colectividades territoriales están implicadas estrategias de colaboración de desarrollo. Destacan en este ámbito los dispositivos de transferencias intergubernamentales destinados a favorecer la igualdad entre las colectividades territoriales. Esto favorece, a través de los múltiples colaboradores, la formación de una clase de entramado de territorios, que es reforzado además por la orientación tomada a favor del desarrollo urbano.

\subsection{Hacia el desarrollo urbano}

Una nueva orientación es impuesta desde principios de los noventa: el objetivo de desarrollo local se desplaza a las ciudades, marcando un cambio en el concepto de desarrollo local de principios de los años setenta que estaba identificado al desarrollo rural y asociado a la noción de país (Georges, 2003). En ruptura con la imagen tradicional, esencialmente rural del alcalde-agricultor, como resultado de las elecciones municipales de 1989, fueron elegidos en varias ciudades francesas los alcaldes-empresarios, decididos a administrar a sus colectividades con los métodos utilizados en su vida profesional anterior.

A menudo también incluía confiar la gestión de ciertos servicios públicos municipales a empresas privadas cuando éstas ofrecían una mejor relación calidad-precios. La elección entre delegar un servicio público o proveerlo directamente por una empresa pública se justificaba en el balance costo-beneficio (Cabrit y Dapogny, 2000).

Esta nueva orientación se inicia con la creación de microregiones, lo cual es particularmente coherente con la idea de un desarrollo endógeno ascendente. Debe subrayarse a este respecto el rol y la influencia que pudieron tener las Cartas Intercomunales de Desarrollo y de Ordenación del Territorio, instituidas en 1983, que si bien su contenido era principalmente jurídico y financiero, estaban basadas en la ideología de la solidaridad, no obstante que era el momento mismo cuando las virtudes de la competencia eran preconizadas en los análisis económicos neoliberales (Drouin, 2006).

El otro aspecto original reside en que las cartas mencionadas representan una invitación a remontar las divisiones habituales para formar un entramado entre municipalidades y el sector privado, orientado a una coproducción del desarrollo local a pesar de que las mentalidades aún estaban marcadas fuertemente por la división entre el sector público y el sector privado. De esta manera, la legislación otorga a las autoridades citadinas un rol de órgano mediador entre los dos sectores, de negociar y debatir con el conjunto de actores socio-económicos, con las asociaciones 
y con la población. Su papel contribuye a hacer penetrar en el sector local nociones nuevas tales como la estrategia de desarrollo, por ejemplo, y, más generalmente, a hacer reflexionar sobre una filosofía de la acción local en su campo de competencia.

De esta manera, hoy la ciudad -el mundo urbano- se afirma expresamente como real polo de desarrollo económico y al mismo tiempo este espacio es reconocido como portador de problemas sociales a resolver. Dentro de este enfoque (Cabannes, 2007) el desarrollo local, se supone, pasa por el desarrollo urbano y particularmente por la creación de un entramado del territorio en el cual la estructura fundamental, el esqueleto, debe estar constituido por una red de ciudades agrupadas en diversas aglomeraciones.

Hasta el primero de enero de 2008, pertenecían a una agrupación intermunicipal con fiscalidad propia 33,638 municipios que representan más de $90 \%$ de los municipios franceses; es decir, estos municipios se asociaron voluntariamente con el objeto de financiar con impuestos directos los servicios públicos que ofrecen en el conjunto de sus territorios. ${ }^{5}$

El objetivo no es realizar oasis de opulencia, sino más bien estructurar el territorio sobre la base de redes descentralizadas apoyadas en primer lugar sobre las grandes metrópolis regionales, también se conformaría por ciudades medianas y pequeñas. La finalidad es reunir en sus redes las potencialidades complementarias, forjar la solidaridad; tal es la idea de fondo que contribuye a definir este enfoque de desarrollo local. Tal lógica no puede hacer abstracción de la competencia que existe entre metrópolis tanto a escala nacional como internacional; el fenómeno concierne en primer lugar a las grandes ciudades con vocación europea que se esfuerzan por posicionarse como ciudades internacionales, ya sea por su apariencia o por el poderío, o imponerse por su función política y cultural.

Los objetivos que suelen pretender numerosas ciudades son diversos, para lograrlo se requieren enormes montos de recursos que sobrepasan a los que requieren normalmente los municipios y necesitan una administración particularmente eficiente. Más la competencia no es solamente entre las grandes ciudades, se establece igualmente entre ciertos municipios, departamentos y regiones; de esta manera, para lograr un desarrollo económico equilibrado del territorio se requiere una concertación o coordinación de acciones locales.

${ }^{5}$ Como ya se dijo, la agrupaciones con fiscalidad propia pueden ser urbanas (hay 16 y agrupan a más de seis millones de habitantes, es la máxima categoría de asociación intermunicipal en el Estado francés,) o comunidad de aglomeración (se registran 174 comunidades de este tipo y agrupan a más de 19 millones de habitantes). 


\subsection{El dilema: descentralización vs centralización fiscal}

Otro elemento importante que ha incidido en la transformación de las finanzas locales es la descentralización, que lógicamente ha engendrado una multiplicación de actores con capacidad de decidir y de administrar (Brunet-Léchenault, 2000). Las consecuencias fiscales de tal proceso no están todavía claramente definidas y la adaptación del poder fiscal local a la nueva situación parece efectuarse de manera totalmente pragmática. ${ }^{6}$ El problema fundamental es la complicación de un sistema político y administrativo que se descentraliza, es decir que se diferencia. Tal fenómeno engendra inevitablemente un sistema que en la medida que se desarrolla puede parecer cada vez más impreciso. La reacción de tal situación puede consistir en tratar de clarificar simplificando, lo cual no siempre es la solución más adecuada.

Desear simplificar el sistema fiscal local puede conducir al retorno de una fiscalidad totalmente dependiente del Estado. Por ejemplo, establecer una cierta dotación de recursos para todo el país, repartirla luego por la administración central entre las colectividades según ciertos criterios bien definidos tiene la ventaja de conferir a la imposición una neutralidad que no se puede tener cuando la decisión es descentralizada (Oates, 1977). Además, la centralización permite la armonización del sistema fiscal pues las reglas de imposición serán las mismas para todos.

De esta manera, la descentralización será compatible con una centralización fiscal que se identificará a una forma de subvención. Los partidarios de tal solución estiman que el objetivo deseable de simplificar al sistema fiscal se ajusta a la necesidad económica de evitar las distorsiones derivadas de la concurrencia y la deslocalización no justificada de empresas, así como de hogares.

La gran desventaja es que de esa manera se suprime la autonomía fiscal de las colectividades territoriales, lo cual se opone a la lógica de la descentralización que supone por esencia una diversificación de medios asociados a una responsabilización de actores en materia financiera, así como en otros campos (Boadway, 2000). Por otro lado, toda reducción de la variedad de fuentes de financiamiento de una colectividad la vuelve más dependiente de las estrategias de sus proveedores de fondos.

La dependencia financiera excesiva respecto a uno de estos proveedores supone una limitación para la autonomía de las autoridades locales que la padecen e impide la responsabilidad fiscal, al encubrir las relaciones fiscales entre los ciudadanos y sus gobiernos. La falta de autonomía, es

\footnotetext{
${ }^{6}$ Según el estudio de Kappeler et al. (2013), la descentralización de ingresos tiene un efecto positivo en la provisión de infraestructura a nivel local, no así en la redistribución de ingresos.
} 
decir la dependencia, conduce a la irresponsabilidad, a la traslación de responsabilidad hacia aquellos de quien se depende.

La cuestión es saber determinar cuál es la solución más pertinente, es decir, la más susceptible de asegurar el bienestar de los ciudadanos, precisamente esto (el bienestar de los ciudadanos) es la única justificación seria de un debate sobre esta materia.

\section{Elemento clave: la autonomía fiscal}

Al igual que la administración pública central, las colectividades territoriales tienen la posibilidad de recurrir al impuesto para financiar sus gastos. Sin embargo, su poder fiscal está limitado en Francia, puesto que no tienen la capacidad de decidir la creación, modificación o supresión de un impuesto. Esta materia es del dominio parlamentario en virtud de que la Constitución de 1958 otorga al parlamento competencia para fijar las reglas relativas a la base, la tasa y la fuente de toda naturaleza de impuestos.

Así pues, el poder fiscal de las colectividades locales francesas se reduce a la posibilidad de votar el monto de impuestos locales, así como las tasas, pero en el marco de límites fijados por la ley y precisados en los reglamentos. Tal capacidad en materia fiscal no es realmente despreciable. Los recursos fiscales, a diferencia del crédito, son definitivos, además de relativamente autónomos. En fin, constituyen una garantía de autonomía para las colectividades tanto de las instituciones financieras como del Estado.

Es importante aclarar que la fiscalidad local ${ }^{7}$ se compone de impuestos directos e indirectos. Los primeros representan las tres cuartas partes del total y entre ellos predominan cuatro impuestos: impuesto de habitación, profesional, sobre los bienes raíces edificados y no edificados. ${ }^{8}$ La fiscalidad directa local no ha sido objeto de una revisión general desde principios de 1970, su obsolescencia ha provocado una desconexión creciente entre las capacidades contributivas de los ciudadanos (propietarios de inmuebles,

\footnotetext{
${ }^{7}$ Un importante sector de la población considera excesiva y obsoleta a la fiscalidad local como puede verse en los siguientes sitios de internet: impots-locaux.net, impots-utiles.com o contribuables. org.

${ }^{8}$ Francia se distingue de otros países por una fiscalidad local muy dependiente de una base inmobiliaria. Los valores catastrales constituyen la base del impuesto sobre bienes raíces y de la tasa de habitación, así como de una fracción de la tasa profesional. Ahora bien, estos valores catastrales han estado durante mucho tiempo subvaluados. El valor de las propiedades no construidas no ha sido revisado desde 1961 y el de las propiedades edificadas desde 1970. Para atenuar los efectos de tal situación se procedió a su actualización por la aplicación de un coeficiente nacional que no ha permitido un acercamiento entre los valores catastrales y los del mercado.

También el parlamento por ley de 1990 decidió proceder a una revisión general de las bases catastrales, las cuales fueron determinadas pero no se aplicaron por miedo a las consecuencias que podía provocar su utilización.
} 
empresas y residentes) y los impuestos pagados, al igual que entre las necesidades de financiamiento de las colectividades locales y la actividad económica ejercida en su territorio (Creusot y Benjamin, 2007).

La importancia de esta problemática se explica porque la autonomía financiera de las colectividades locales se ha retomado hoy en el cuadro de los debates relativos a la libre administración de éstas, con motivo de la revisión constitucional del 2003. La cuestión de la autonomía fiscal constituye el punto central de tales discusiones.

\subsection{El marco normativo de la autonomía fiscal}

Las preguntas relativas a las relaciones que pueden mantener la autonomía fiscal y el principio de libre administración de las colectividades territoriales conducen, en primer lugar, a interesarse en las repuestas a nivel constitucional. Se puede constatar que todas las decisiones sobre la materia se orientan a la misma conclusión: se busca preservar la libre administración. Para ello, en las modificaciones a los impuestos locales que se plantean actualmente se dice en un reporte de la Délégation interministérielle à l'aménagement du territoire et à l'attractivité (DATAR) que no se busca disminuir los recursos globales de las colectividades locales ni recortar sus recursos fiscales al punto de dificultar su libre administración, pero no se aportan los elementos precisos que permitan determinar un umbral que no se pueda sobrepasar (DATAR, 2003).

Se puede comprender que encarar una posición que no define qué es el umbral de los ingresos fiscales, por debajo del cual sería cuestionado el principio constitucional de la libre administración de las colectividades locales, conduce a buscar la respuesta no en el terreno jurisdiccional, sino en el de la política.

La modificación constitucional realizada en 2003 extiende el campo de competencias de las colectividades territoriales e introduce la posibilidad para ellas de derogar, a título experimental y por un objeto y una duración limitada, las disposiciones legislativas o reglamentarias que restrinjan el ejercicio de sus competencias, esto amplía la autonomía financiera local; aunque es importante señalar que a la fecha ninguna colectividad ha usado esta facultad (Laurent, 2009). La Ley constitucional 2003-276 en el artículo 72-2 dispone, por un lado, que las colectividades territoriales pueden recibir todo o parte del producto de los impuestos de cualquier naturaleza y que la ley puede autorizarlas a fijar la base imponible y la tasa del gravamen en los límites que ella determina.

Por otro lado, también señala que los ingresos fiscales y los otros recursos propios de las colectividades territoriales deberán representar para cada categoría una parte importante del total de sus recursos. 
En fin, que toda transferencia de competencias entre la administración central y las colectividades territoriales se acompañe de los recursos correspondientes y que toda creación o extensión de competencias, que tenga como consecuencia un incremento en los gastos de las colectividades territoriales, sea acompañada de recursos determinados por la ley. Se puede estimar que este dispositivo tiene por objetivo que las comunidades locales puedan autofinanciarse a fin de asegurar su libre administración; lo que no implica autonomía de decisión.

La Ley Orgánica del 29 de julio de 2004 define los elementos que permiten determinar la noción de autonomía fiscal y, por consecuencia, lo que se entiende por recursos propios. Entran en esta categoría todos los impuestos sobre los cuales los gobiernos locales tienen un grado relevante de responsabilidad, tanto para determinar los elementos esenciales del tributo, como son la base, la tasa o la tarifa, o en los que ellos determinan la tasa, o una parte local de la base gravable. Figuran igualmente los ingresos por servicios prestados, los productos de las propiedades públicas, las participaciones de urbanismo, los productos financieros y las donaciones y legados. No son incluidos dentro de esta categoría los préstamos e ingresos de tesorería ni las subvenciones o dotaciones provenientes del Estado o de otras colectividades.

La garantía de la autonomía financiera es reconocida no para las colectividades locales tomadas individualmente sino para sus categorías, es decir, los municipios, departamentos y regiones. Se excluye por tanto a las estructuras intercomunales.

Hay otra concepción diferente en cuanto a la autonomía financiera local, que propone reducir la autonomía fiscal de las colectividades territoriales haciendo desaparecer progresivamente los principales impuestos locales, sustituyéndolos por compensaciones las cuales finalmente se transformarían en dotaciones, que deberán ser suficientes para asegurar la libre administración de las colectividades territoriales. Según este enfoque, la libertad debe estar en la elección de los gastos, sin importar quién determine los impuestos para cubrirlos, en este sentido habrá autonomía local sin autonomía fiscal. Se trata de dos concepciones de la descentralización y del Estado que están a debate, razón por la cual resulta indispensable una lectura política.

En efecto, si se admite que el origen y la fuerza de todo poder político es determinado en gran parte por la posesión de un poder fiscal autónomo, no por la simple gestión de medios financieros procurados y concedidos por otros, parece natural que la cuestión de la libre administración viene a cristalizar alrededor de un debate centrado principalmente en lo que se entiende por poder fiscal local. 
En los últimos años se observa en Francia una tendencia a reducir la autonomía fiscal de las colectividades territoriales al desaparecer progresivamente los impuestos directos locales, en los que las colectividades territoriales tienen una relativa autonomía de decisión, como es el caso de la tasa profesional que en 2010 se ha anunciado su desaparición para reducir la carga fiscal sobre la inversión y la innovación de las empresas. Así, se ha optado por hacer prevalecer la autonomía de gestión sobre la autonomía de decisión. Se considera (Laurent, 2009) que para invertir esta tendencia se requiere una reforma fiscal amplia que se oriente no solamente a la creación de nuevos impuestos rentables para las colectividades locales, sino también por la posibilidad de que ellas decidan las tasas y eventualmente las bases gravables de esos impuestos; además eliminar la facultad del Estado de negociar con ciertos sectores de la población la desgravación de algunos impuestos locales. Luego pues, esto es de envergadura.

A fin de comprender la magnitud del reto, conviene agregar que tal debate y tal vía debe ser llevado a cabo en el marco de la Unión Europea. De hecho, el reto tiene esencia fundamentalmente política en la medida que se introduce el problema de la organización del poder fiscal.

\subsection{Los límites del poder fiscal}

Al partir de la objeción política fundamental es conveniente no conferir un poder fiscal a las colectividades locales tan amplio que pueda provocar la fragmentación de la red financiera local transformándola en múltiples feudos (Albert y Aglae, 2003).

Históricamente la fiscalidad ha permitido la construcción de Estados más sólidos. En la actualidad, el proceso de descentralización podría ser la premisa hacia una nueva Edad Media fiscal en el seno de las sociedades contemporáneas.

Por otro lado, es conveniente subrayar que la conducción de un sistema local diversificado y complejo se vuelve cada vez más difícil en la medida que se incrementa la autonomía de los actores, la multiplicación de éstos, incluso la opacidad de sus relaciones.

En total y desde un punto de vista general, el problema esencial, al cual se enfrenta la cuestión de la autonomía fiscal de las colectividades locales, es la complejidad de un sistema administrativo y político que al descentralizarse $-y$ por consecuencia, y sobre todo disponer de medios financieros suficientes- no puede más que diversificarse, con lo que su control es muy complejo.

Es necesario señalar que la observación de las reformas adoptadas recientemente en Francia, en materia de fiscalidad local, hace evidente que 
no se intenta encarar la complejidad del medio local por medio de una red diversificada de lugares y de procedimientos de control; las reformas van en sentido contrario ya que han optado por un control desde arriba, por un control central (Creusot y Benjamin, 2007).

Tal evolución, que limita la autonomía fiscal local, restringe la variedad de recursos autónomos de financiamiento, lo que convierte a las colectividades locales necesariamente dependientes de estrategias de su principal proveedor de fondos que es la administración central y que lleva a reforzar la capacidad de regulación de éste, con lo que no solamente será el sostén como en otros tiempos sino también se convertirá en el órgano superior de control.

Resulta indispensable no confundir la autonomía de gestión y la autonomía de decisión. Se puede decir que si son suficientes y globalizadas las transferencias financieras del Estado es posible que las comunidades locales puedan elegir alternativas en particular de gestión, pero no tendrán un real poder de decisión, ya que para ello necesitan un dominio al menos relativo de fuentes de financiamiento. En otros términos, las colectividades locales tendrán una verdadera capacidad de decisión solamente si su autonomía de gestión está asociada a un poder fiscal.

Precisamente en este terreno se juega el futuro de la descentralización. Las posibilidades son que las colectividades territoriales tengan cierta autonomía o bien, sean simples espacios de gestión.

En los últimos 30 años las comunidades locales no se han limitado exclusivamente a ofrecer servicios y a tratar de satisfacer las necesidades de sus administrados, sino que también han sido las encargadas de elegir gastos e ingresos y de manejar el crédito. Esta es la razón por la que la autonomía fiscal se ha vuelto a lo largo del tiempo el elemento crucial del debate relativo a la libre administración de las colectividades territoriales (Creusot y Benjamin, 2007).

La teoría fiscal considera a la fiscalidad local como una vía de acceso privilegiada a una responsabilización de la gestión pública y un elemento clave de la democracia de proximidad, ya que permite acercar los centros de decisión a los ciudadanos, lo cual se puede traducir en mecanismos más transparentes, más democráticos, más rápidos y eficaces en los procesos de toma de decisiones y en la implementación de las políticas. Así, por ejemplo, las autoridades tendrán que asumir ante sus electores que no se puede disponer de un nivel elevado de servicios públicos y a la vez de una carga fiscal inferior a la media.

Si fuera ciertamente indispensable reconsiderar el sistema fiscal local, incluso hasta convenir la supresión de ciertos impuestos, eso no implica que sea recomendable que recurran al financiamiento del Estado, pues se 
corre el peligro de que la relación democrática entre los ciudadanos y sus gobernantes se sustituya por la reivindicación frente al gobierno central.

\subsection{Escenarios del futuro: el debate}

El proceso actual de descentralización en Francia suscita un cierto número de temores que se pueden reagrupar en dos grandes categorías: los temores de orden político se expresan por el miedo a un desmembramiento del Estado; los temores de orden más directamente financiero por el riesgo de una explosión de impuestos locales ligada a la extensión de competencias de las colectividades territoriales prevista por la ley relativa a las responsabilidades locales (Piron, 2006); además de otros posibles efectos de largo plazo como sería la relación inversa entre la cantidad de ingresos recaudados por las colectividades locales y el tamaño del gobierno (Ashworth et al., 2013), y sus posibles efectos negativos en la provisión de bienes públicos locales (Breuille y Zanaj, 2013).

Existe consenso en cuanto a que el nuevo proceso de descentralización que se inicia no constituye una simple respuesta a una crisis de las finanzas del Estado, sino que es parte de una reforma financiera de éste, que necesita una reorganización del sector público en su conjunto, de sus estructuras y de su gestión. Desde luego la causa es financiera pero persigue objetivos más amplios.

Es decir, en este contexto, no se trata de oponer el Estado a las colectividades locales sino más bien de adoptar una concepción integradora. Excluir al Estado sería amenazar la unidad de la sociedad, arriesgar su fragmentación y significaría prácticamente la entrada a una nueva Edad Media (Bouvier, 2007). Excluir a las colectividades locales sería el regreso al centralismo y a la rigidez cuando la flexibilidad de las instituciones es, más que nunca, necesaria en el seno de un mundo inestable y fluctuante. Se considera que las regiones deberían ser los espacios de integración, los lugares privilegiados de coherencia entre las acciones del Estado y de las colectividades locales.

Así pues, el problema de fondo que falta resolver ahora no es estrictamente financiero, se trata de organizar y asumir la dirección de un sistema complejo así como de evitar un desarrollo incontrolado de éste.

Desde el punto de vista de las finanzas públicas, numerosos autores (Bouvier, 2007; Creusot y Benjamin, 2007; Laurent, 2009; Buisson, 2009) consideran indispensable que funcione un órgano paritario que reúna representantes del Estado y de las colectividades territoriales, cuya tarea sea regular por concertación la evolución de los recursos, de los gastos públicos y de las subvenciones con fines redistributivos a instituir. 
Este órgano tendría la tarea de coordinar, armonizar, incluso negociar, de manera plurianual la evolución proyectada en cuanto a lo que concierne a las libertades fiscales de las comunidades como las políticas financieras generales relativas a los gastos y a los recursos. Este cuerpo ${ }^{9}$ se encargaría de asegurar que el nuevo reparto de funciones (transportes, carreteras, acción social, habitación, etc.) se haga con las compensaciones financieras suficientes del Estado y sin una explosión de la fiscalidad nacional o local.

Como se observa, la tendencia es que las reformas y las normas fiscales se inscriban y desarrollen en un marco de decisión y de gestión global para no perder el control del sistema financiero público. Hay coincidencia en cuanto a que la gestión pública general y de las finanzas locales en particular se debe orientar hacia una racionalización y un dominio repartido de ingresos y de gastos públicos entre los actores.

En este contexto, un tema de gran relevancia resulta ser la elección del esquema más adecuado de reparto de fuentes impositivas entre los diferentes niveles de gobierno. Algunos tratadistas proponen la organización descentralizada del Estado, pero nada más en la estructura y no en su dinámica. La solución propuesta es la especialización fiscal, la cual es sostenida por algunos por simple pragmatismo y por otros para aprovechar las ventajas del federalismo fiscal.

Tal proposición se basa en la crítica que pareciera fundada de que la superposición de impuestos locales engendra múltiples inconvenientes tales como que una misma base impositiva sea utilizada por dos niveles diferentes de colectividades, la opacidad de las decisiones fiscales para los contribuyentes. Según sus partidarios, la especialización fiscal o la utilización de un impuesto local por nivel de colectividad, tendrá el mérito de clarificar, simplificar, hacer más coherente el sistema fiscal y evitar un incremento de la presión fiscal.

Esta dirección puede parecer correcta en la medida que propone una imagen ordenada y racional de la fiscalidad local compatible con una organización descentralizada. Sin embargo, este sistema obliga a restringir las capacidades de iniciativa y la creatividad de las colectividades, las hará rígidas. Por lo tanto, atenta contra la libertad de elección de sus impuestos, que en el contexto internacional de hoy tendrá seguramente el efecto de disminuir su capacidad de responder al papel que deberían desempeñar las colectividades.

${ }^{9}$ En 2006 se instituyó en Francia la Conferencia Nacional de Finanzas Públicas, que es un dispositivo para regular el sistema financiero público, aunque concierne exclusivamente al gasto público. Para varios autores el ideal es que se convierta en un lugar de regulación concertada de gastos y de ingresos públicos. En 2007 se creó la Conferencia Nacional de Ejecutivos, institución que tiene la misión de hacer participar a las colectividades territoriales en la elaboración de normas que le conciernen así como en la reforma de la fiscalidad local que se plantea en la Unión Europea. 
Desde luego, atribuir un impuesto único y específico a cada uno de los componentes del sistema local no sería conferir un verdadero poder fiscal. Así, para algunos autores (Bouvier et al., 2008) este poder sería real si descansara en una pluralidad de impuestos, de los cuales todos o solamente una parte pueden ser específicos.

Este modelo de especialización que se diferencia de la concepción del impuesto único atribuido a cada categoría de colectividades es coherente con un sistema complejo de funciones diferenciadas, tal como es el sistema fiscal.

$\mathrm{Si}$ se quieren conservar las ventajas a que da lugar el funcionamiento según esta lógica del sistema, más que una repartición voluntarista de impuestos será preferible promover la coordinación de los diferentes niveles de gobierno, es decir, dejar desarrollar la iniciativa local y tratar al mismo tiempo de compensar las inigualdades con la instalación de un sistema de reparto de recursos.

De esta manera podrían coexistir la independencia y la coordinación, así como la competencia y la solidaridad, aunque pueda parecer paradójico; se trata de que sea un principio inherente y esencial a todo sistema realmente descentralizado y en el que la restricción no puede más que limitar y debilitar a sus concurrentes (Bouvier, 2007). Con la coordinación se puede aprovechar la fuerza del sistema, su capacidad de responder a los retos que se le presenten, así como aprovechar la variedad de medios de que disponga, y adecuarse a sus circunstancias.

Hoy en día se observa que una cierta especialización de los diferentes niveles de colectividades combinada con una variedad de instrumentos fiscales está presente en el sistema local francés, particularmente a partir del desarrollo de agrupamientos de municipios con fiscalidad propia y específica. Lo que podría ser útil es una mayor especialización fiscal desarrollada de manera voluntaria; adaptar los mecanismos existentes, perfeccionarlos, e incluso, buscar una integración fiscal más adecuada al nivel de espacios de decisión más grandes, constituyen algunas de las soluciones más adaptadas a la dinámica del sistema.

\section{Conclusiones}

Si se considera solamente el análisis de la evolución en el tiempo de los aspectos estructurales de las finanzas locales no se observan cambios fundamentales en las finanzas locales francesas. A pesar de las reformas de los últimos 30 años, la fiscalidad permanece similar a la de antes. Las fuentes de recursos siguen siendo los impuestos, las transferencias y los créditos; aunque las técnicas utilizadas para su obtención se han vuelto más com- 
plicadas. El aumento de los presupuestos locales y sus cambios de orientación en realidad han estado presentes desde hace mucho tiempo.

Los grandes cambios de las estructuras fiscales locales se deben a la descentralización, que ha forjado una reformulación de los objetivos y de los retos de las finanzas locales.

El elemento nuevo es la descentralización bajo el principio de autonomía de decisión de las comunidades locales, lo que ha podido engendrar un incremento considerable de acciones locales y, por consecuencia, de sus presupuestos, así como un cambio de sus objetivos. Su importancia no es sólo desde el punto de vista de la prestación de un conjunto de servicios sino también del desarrollo económico, hoy en día juegan un papel clave en el conjunto de la inversión pública, con lo que se subraya la importancia de la actividad local en términos macroeconómicos.

Con el nuevo rol de las colectividades locales se pretende acercar lo máximo posible la provisión de los servicios y los centros de toma de decisiones y de poder hacia los ciudadanos, es decir, mejorar los canales de participación ciudadana, favorecer la gestión de tipo transversal evitando los estancamientos y las situaciones de competencia entre distintas administraciones.

Es importante subrayar que el surgimiento del poder local se ha dado en un contexto económico muy específico, puesto que Francia ha pasado de un periodo de crecimiento a uno de crisis. Por otro lado, han influido los análisis económicos neoliberales. Al mismo tiempo, la Unión Europea ha entrado igualmente a una nueva fase de su desarrollo con la introducción de la moneda única, la entrada de nuevos países y la crisis financiera del 2010. Sobre el terreno de métodos y técnicas, las tecnologías se han adaptado a un tipo de organización más compleja para responder a los nuevos retos que enfrenta.

Desde este punto de vista, como se señaló anteriormente, si los instrumentos financieros de las colectividades territoriales permanecen igual, entonces sus mecanismos tienen que ser modificados para evitar un incremento explosivo en los impuestos locales, el cual derive de la extensión de competencias de las colectividades territoriales. Una de las respuestas a esta problemática sería la coordinación de los diferentes niveles de gobierno, el fin último deberá ser el bienestar de los ciudadanos; esta respuesta, a pesar de tratarse de realidades muy diferentes, es válida para el caso mexicano, en el cual, además, por ser un Estado federal, resulta incuestionable la necesidad de que las entidades federativas cuenten con autonomía de decisión. 


\section{Bibliografía}

Albert, Jean-Luc y Luc Saïdj (2009), Finances publiques, Éditions Dalloz, Paris.

Albert, Jean-Luc y Marie-Joseph Aglae (2003), Fiscalité \& développement, Éditions Cujas, Paris.

Ashworth, John, Emma Galli y Fabio Padovano (2013), "Decentralization as a constraint to Leviathan: a panel cointegration analysis", Public Choice, 156 (3-4), Springer, New York, pp. 491-516.

Aubouin, Michel y Jean-Christophe Moraud (2006), Administrer les territoires, Dexia Crédit Local, Paris.

Boadway, Robin (2000), "Recent developments in the economics of federalism", en Lazar Harvey (ed.), Toward a new mission statement for canadian fiscal federalism, McGill-Queen's University Press, Montreal-Kingston, pp. 41-78.

Boeuf, Jean-Luc y Manuela Magnan (2009), Les collectivités territoriales et la décentralisation, La Documentation Française, Paris.

Bouvier, Michel (2007), Introduction au droit fiscal général et à la théorie de L'impôt, Librairie Générale de Droit et de JurisprudenceMontchrestien, Paris.

Bouvier, Michel, Marie-Christine Esclassan y Jean-Pierre Lassale (2008), Finances publiques, LGDJ, Paris.

Breuille, Marie-Lure y Skerdilajda Zanaj (2013), "Mergers in fiscal federalism”, Journal of Public Economics, vol. 105, Elsevier, Amsterdam, pp. 11-22.

Brunet-Léchenault, Claudette (2000), La décentralisation et le citoyen, Les éditions des Journaux Officiels de la République Française, Paris.

Buisson, Jacques (2009), Finances publiques, Éditions Dalloz, Paris.

Cabannes, Xavier (2007), Finances publiques, LMD Collection, Paris.

Cabrit, Alain y Bernard Dapogny (2000), L'Élu municipal, Éditions du Puits Fleuri, Paris. 
Cassette, Aurelie, Etienne Farvaque, y Jerome Hericourt (2013), “Tworound elections, one-round determinants? Evidence from the french municipal elections", Public Choice, 156 (3-4), Springer, New York, pp. 563-591.

Creusot, Sébastien y Olivier Benjamin (2007), Le financement des nouvelles compétences des collectivités locales, LGDJ, Paris.

DATAR (Délégation interministérielle à l'aménagement du territoire et à l'attractivité) (2003), Une nouvelle politique de développement des territoires pour la France, Comité stratégique de la DATAR, Paris.

Drouin, Vincent (2006), Fantassins de la République, nos 500000 conseillers municipaux, éditions Autrement, Paris.

Fabbrini, Federico (2013), "The fiscal compact, the 'Golden Rule' and the paradox of european federalism", Boston College International \& Comparative Law Review, 36 (1), Boston College Law School, Boston, pp. 1-38.

Facchini, Francois y Mickael Melki (2013) "Efficient government size: France in the 20th century", European Journal of Political Economy, 31, Elsevier, Amsterdam, pp. 1-14.

Faure, Alain y Anne-Cécile Doiullet (2005), L'action publique et la question territoriale, Presses Universitaires de Grenoble, Grenoble.

Georges, Dmitri (2003), La république décentralisée, L'Harmattan, Paris.

Gevart, Pierre (2006), Comprendre les enjeux de la décentralisation, Éditions l'Etudiant, Paris.

Gouttebel, Jean-Yves (2010), Le consultant et le développement territorial, Economica, Paris.

Greffe, Xavier (2005), La décentralisation, Éditions La Découverte, Paris.

INSÉÉ (Institut National de la Statistique et des Études Économiques) (2009), Comptes nationaux, Ministère de l'intérieur de l'outreMer et des collectivités territoriales, Paris.

Kappeler, Andreas, Albert Sole-Olle, Stephan Andreas y Timo Vaeliae (2013), "Does fiscal decentralization foster regional investment 
in productive infrastructure?", European Journal of Political Economy, 31, Elsevier, Amsterdam, pp.15-25.

Laurent, Philippe (2009), Décentralisation: en finir avec les idées reçues, LGDJ, Paris.

Oates, Wallace (1977), Federalismo fiscal, Instituto de Estudios de Administración Local, Madrid.

Piron, Michel (2006), Gouverner en France: quel équilibre territorial des pouvoirs?, Les Éditions des journaux officiels, Paris.

Teyssier, Arnaud (2006), La décentralisation en mouvement, Ministère de l'Intérieur et de l'Aménagement du Territoire, Paris.

Tulard, Marie-José (2006), La coopération décentralisée, LGDJ, Paris.

Recibido: 2 de febrero de 2011. Reenviado: 14 de noviembre de 2012.

Aceptado: 18 de abril de 2013.

Marcela Astudillo-Moya. Mexicana, doctora en administración pública por la Universidad Nacional Autónoma de México. Actualmente es investigadora en el Instituto de Investigaciones Económicas de la misma universidad y profesora en la Facultad de Ingeniería, así como tutora en los programas de posgrado de la Facultad de Ciencias Políticas y Sociales y de la Facultad de Economía. Es miembro del Sistema Nacional de Investigadores, nivel I. Sus líneas de investigación actuales son federalismo fiscal y haciendas locales. Entre sus últimas publicaciones destacan: "Descentralización fiscal y distribución de la renta regional en Francia y en México", Eseconomía Revista de Estudios Económicos, Tecnológicos y Sociales del Mundo Contemporáneo, VII (35), Instituto Politécnico Nacional, México, pp. 37-65 (2012); en coautoría, "Algunas consideraciones sobre el impuesto predial. Una referencia al caso de México", INCEPTUM Revista de Investigación en Ciencias de la Administración, VII (12), Ininee, México, pp. 295-314 (2012); y Fundamentos de economía, unam-Probooks, México, 188 pp. (2012). 